\title{
TFEB activation in macrophages attenuates postmyocardial infarction ventricular dysfunction independently of ATG5-mediated autophagy
}

\author{
Ali Javaheri,, Geetika Bajpai, ${ }^{1}$ Antonino Picataggi," Smrithi Mani, ${ }^{1}$ Layla Foroughi, ${ }^{1}$ Hosannah Evie, ${ }^{1}$ \\ Attila Kovacs, ${ }^{1}$ Carla J. Weinheimer, ${ }^{1}$ Krzystztof Hyrc, ${ }^{2}$ Qingli Xiao, ${ }^{3}$ Andrea Ballabio, ${ }^{4,5,6,7}$ Jin-Moo Lee, ${ }^{3}$ \\ Scot J. Matkovich, ${ }^{1}$ Babak Razani, ${ }^{1,8}$ Joel D. Schilling, ${ }^{1}$ Kory J. Lavine, ${ }^{1}$ and Abhinav Diwan ${ }^{1,8}$ \\ ${ }^{1}$ Cardiovascular Division and Center for Cardiovascular Research, Department of Medicine, ${ }^{2}$ Alafi Neuroimaging Laboratory, \\ and ${ }^{3}$ Hope Center for Neurological Disorders, Washington University School of Medicine, Saint Louis, Missouri, USA. \\ ${ }^{4}$ Telethon Institute of Genetics and Medicine, Pozzuoli, Italy. ${ }^{5}$ Medical Genetics, Department of Medical and Translational \\ Sciences, Federico II University, Naples, Italy. 'Jan and Dan Duncan Neurological Research Institute, Texas Children's \\ Hospital, Houston, Texas, USA. 'Department of Molecular and Human Genetics, Baylor College of Medicine, Houston, \\ Texas, USA. ${ }^{8}$ John Cochran Veterans Affairs Medical Center, Saint Louis, Missouri, USA.
}

Lysosomes are at the epicenter of cellular processes critical for inflammasome activation in macrophages. Inflammasome activation and IL-1 $\beta$ secretion are implicated in myocardial infarction (MI) and resultant heart failure; however, little is known about how macrophage lysosomes regulate these processes. In mice subjected to cardiac ischemia/reperfusion (IR) injury and humans with ischemic cardiomyopathy, we observed evidence of lysosomal impairment in macrophages. Inducible macrophage-specific overexpression of transcription factor EB (TFEB), a master regulator of lysosome biogenesis (M $\phi$-TFEB), attenuated postinfarction remodeling, decreased abundance of proinflammatory macrophages, and reduced levels of myocardial IL-1ß compared with controls. Surprisingly, neither inflammasome suppression nor $\mathrm{M} \phi$-TFEB-mediated attenuation of postinfarction myocardial dysfunction required intact ATC5-dependent macroautophagy (hereafter termed "autophagy"). RNA-seq of flow-sorted macrophages postinfarction revealed that $M \phi$-TFEB upregulated key targets involved in lysosomal lipid metabolism. Specifically, inhibition of the TFEB target, lysosomal acid lipase, in vivo abrogated the beneficial effect of $M \phi$-TFEB on postinfarction ventricular function. Thus, TFEB reprograms macrophage lysosomal lipid metabolism to attenuate remodeling after IR, suggesting an alternative paradigm whereby lysosome function affects inflammation.

Conflict of interest: The authors have declared that no conflict of interest exists.

Copyright: () 2019, American Society for Clinical Investigation.

Submitted: January 9, 2019

Accepted: September 25, 2019

Published: November 1, 2019

Reference information: /CI Insight. 2019;4(21):e127312.

https://doi.org/10.1172/jici.

insight.127312.

\section{Introduction}

Macrophages are a key mediator of disease progression in coronary artery disease, atherosclerosis, myocardial infarction (MI), and heart failure (1-6). After MI, bone marrow and splenic monocytes are recruited to the myocardium via chemokine receptor 2 (CCR2) where they differentiate into macrophages that affect efferocytosis of dead cells (7). CCR2 ${ }^{+}$macrophages have also been implicated in inflammatory collateral tissue injury, leading to left ventricular (LV) dysfunction (8-10). Understanding the cellular mechanisms that attenuate proinflammatory actions of macrophages while promoting reparative functions under conditions of sterile inflammation is an important area of scientific inquiry, with broad applicability to myocardial injury responses and other inflammatory conditions.

One of the central events coordinated by CCR2 $2^{+}$monocytes and macrophages is secretion of the cytokine IL-1 $\beta$ (11), which has been implicated as a therapeutic target in both atherosclerosis (12) and after cardiac ischemia/reperfusion (IR) injury to prevent heart failure $(13,14)$. The lysosomal degradative process of macroautophagy (hereafter termed "autophagy") has been ascribed critical and seemingly paradoxical roles in regulating IL-1 $\beta$ secretion and the myocardial response to IR. In terms of inflammasome activation, autophagy is generally thought to suppress IL-1 $\beta$ production by promoting the turnover of damaged 
mitochondria and reducing ROS, which can leak DNA into the cytosol and thus serve as a stimulus for inflammasome activation (15-18). Paradoxically, autophagy also plays a role in unconventional secretion of IL-1 $\beta$, a mechanism that has now been demonstrated in macrophages and neutrophils $(15,19,20)$.

Analogous to the multiple competing mechanisms by which autophagy regulates IL-1 $\beta$, autophagy has been implicated in both promoting and attenuating myocardial IR injury. Initial studies suggested that oxidative stress and IR injury activated myocardial autophagy and that Becn1 (encoding BECLIN-1, which is required for autophagic initiation) haploinsufficient mice were resistant to IR (21). More recently, we found that reperfusion following ischemia elevated myocardial BECLIN-1 protein, which suppresses activation of transcription factor EB (TFEB) (22), a master regulator of autophagy and lysosomal biogenesis $(23,24)$, with resultant impaired autophagic flux (25). Accordingly, intermittent fasting stimulated nuclear translocation and activation of TFEB and attenuated myocardial IR injury with decreased infarct size, in a manner that depended on an intact autophagy-lysosome pathway (26). In addition, overexpression of macrophage TFEB attenuates inflammasome activation and IL- $1 \beta$ secretion, as well as atherosclerosis (27). An additional challenge confounding the potential role of TFEB in innate immune regulation is that TFEB and its related basic loop-helix family member TFE3 are required for innate immune activation (28). Clarifying the role of TFEB in inflammation and myocardial injury is of great significance, especially given the tremendous interest in the cardiovascular community to leverage the autophagy-lysosome system in heart failure and atherosclerosis therapeutics $(27,29)$.

These aforementioned studies raise critical, unanswered questions regarding the role of macrophage autophagy and TFEB in myocardial IR injury. First, we asked whether macrophage autophagy-lysosome dysfunction occurs after IR injury and in ischemic cardiomyopathy and, if so, does restoring autophagy and lysosomal function by expressing TFEB attenuate injury after IR and inflammation. Based on these questions, we explored the mechanisms whereby macrophage TFEB affects ventricular function after IR and myocardial remodeling in which both inflammasome activation and autophagy have been implicated. Our findings were consistent with lysosomal lipolysis, not autophagy per se, as a critical mechanism by which TFEB is immunomodulatory and may suppress myocardial injury after IR.

\section{Results}

The macrophage autophagy-lysosome functional compartment is compromised after $I / R$ and in the failing myocardi$u m$. Activation and proliferation of resident cardiac macrophages and recruitment of circulating monocytes and their differentiation to macrophages drive a major component of the cellular inflammatory response in the reperfused myocardium, early in the period after $\mathrm{MI}(8,30)$. In addition, multiple risk factors for heart failure, including diabetes mellitus and coronary artery disease, are associated with autophagic impairment in macrophages $(27,31)$, suggesting that alterations in macrophage autophagy may be a persistent phenotype of ischemic cardiomyopathy in humans. To test this hypothesis, we performed costaining for p62, an autophagy adaptor and substrate protein, and the macrophage marker CD68 in donor heart tissue versus heart tissue obtained from patients with ischemic cardiomyopathy. In heart tissue from patients with ischemic cardiomyopathy, we observed a statistically significant increase in p62 positivity in macrophages (Figure 1, A and B). These results were consistent with the hypothesis that human macrophages exhibit autophagic impairment in the setting of ischemic cardiomyopathy.

In prior studies, activation of macrophages by inflammatory stimuli, such as LPS (28) and thioglycollate, and differentiation of monocytes to macrophages (32) has been observed to result in transcriptional induction of lysosome biogenesis. Accordingly, to determine whether lysosomal abundance is affected in cardiac macrophages in the inflammatory milieu after MI, we subjected adult (8-week-old) male C57BL6 mice to closed-chest myocardial IR injury or the sham procedure and prepared cardiac cell suspension for flow cytometry. Closed-chest IR was modeled by reversible occlusion of the left anterior descending (LAD) coronary artery for 90 minutes, 2 weeks after thoracotomy for implantation of the suture to permit healing and circumvent the inflammatory response associated with thoracotomy $(33,34)$. We initially chose a time point of 4 days after MI, in which we observed a significant increase in CCR2 ${ }^{+}$macrophages compared with sham-operated mice (Supplemental Figure 1, A and B; supplemental material available online with this article; https://doi.org/10.1172/jci.insight.127312DS1). CCR2 ${ }^{+}$macrophages remained elevated for 7 days after IR (Supplemental Figure 1C) compared with sham, which is also consistent with the peak observed at 3 days in open-chest models of MI (reviewed in ref. 4). To identify acidified lysosomes, LysoTracker Red staining was assessed in cardiac monocytes and macrophages (Figure 2A), and noted to be 
approximately 4-fold higher in macrophages compared with monocytes in the sham heart (Figure 2, B and C), likely reflecting an induction of the lysosome biogenesis program on differentiation from monocytes to macrophages in the myocardium. Interestingly, this increased LysoTracker staining was significantly attenuated in macrophages obtained from IR-injured myocardium (Figure 2C), despite the preceding myocardial injury. This finding indicates either a deficiency of acidified macrophage lysosomes or in lysosome number in the setting of stress, despite the increase in inflammatory stimuli that are released from injured and dead cells in the setting after IR (4).

We next sought to determine whether the relative decrease in lysosomal abundance was associated with a subsequent autophagic impairment in macrophages in the heart after IR by performing closed-chest IR injury in CAG-RFP-EGFP-LC3 mice that express the autophagosome protein LC3 fused in tandem to both RFP and EGFP, as a reporter of autophagic flux (35). In prior experiments with LysoTracker that were conducted at day 4 , mostly CCR2 ${ }^{+}$macrophages were typically present. We hypothesized that the more proinflammatory CCR2 ${ }^{+}$macrophages may exhibit differences in autophagy compared with the more reparative CCR2- macrophages, and thus performed flow cytometry in CAG-RFP-EGFP-LC3 mice 7 days after IR, in which both $\mathrm{CCR} 2^{+}$and $\mathrm{CCR} 2^{-}$macrophages are more evenly distributed. In cells bearing this reporter, GFP is quenched in an acidified environment, and therefore, the RFP signal is predominant when LC3 enters autolysosomes (35). After closed-chest IR, we identified Ly6C ${ }^{\text {hi }}$ CD $64^{\text {hi }}$ macrophages that were further subclassified based on CCR2 expression prior to examination of the RFP-EGFP-LC3 signal. Compared with sham-operated animals, we observed a reduction in relative intensity of the RFP signal in macrophages after IR (Figure 2, D and E), consistent with the reduced abundance of autolysosomes in macrophages. Using the GFP/RFP signal intensity ratio as a metric of the relative abundance of autophagosomes/autolysosomes, we noted that $\mathrm{CCR} 2^{+}$macrophages exhibited increased abundance of autophagosomes (relative to autolysosomes) compared with CCR2- macrophages. Importantly, after IR, although there was not a significant change in the relative abundance of autophagosomes/autolysosomes in CCR2 ${ }^{+}$ macrophages, CCR2- macrophages demonstrated a significant increase, suggesting impaired autophagosome clearance (i.e., autophagic impairment) in CCR2- macrophages specifically after IR (Figure 2, D and E). Taken together, these findings were consistent with a hypothesis of relative deficiency of the autophagy lysosome pathway in ischemic cardiomyopathy and after IR, which was likely secondary to a relative deficiency of acidified lysosomes.

To investigate mechanisms whereby MI may lead to macrophage autophagic impairment, we examined autophagic flux in peritoneal macrophages (pMACs) under normoxic conditions or after hypoxia/reoxygenation (Supplemental Figure 2, A-C). Under normoxic conditions, control macrophages exhibited intact autophagic flux, as evidenced by accumulation of both p62 and LC3-II (a marker of autophagosome abundance) upon treatment with a lysosome acidification inhibitor (bafilomycin A1), in comparison with either ATG5-deficient macrophages or macrophages subject to hypoxia/reoxygenation stress. These experiments demonstrated that hypoxia/reoxygenation is a sufficient stress to impair macrophage autophagic flux.

Activation of the macrophage autophagy-lysosome pathway prevents remodeling after $I R$. To overcome the relative lysosomal insufficiency in macrophages after MI, we induced autophagy and lysosomal biogenesis by selectively overexpressing TFEB in macrophages (27). Toward this end, we generated transgenic mice that specifically overexpress TFEB in macrophages. We crossed a previously generated Tfeb-transgenic mouse line designed for conditional overexpression that carries TFEB-3xFlag expression cassette under the control of a strong chicken $\beta$-actin (CAG) promoter followed by a transcriptional terminator that is flanked by lox sites (23) with a mouse line that expresses the macrophage-specific $\mathrm{Cx} 3 \mathrm{Cr} 1$ erCre (termed "M $\phi$-TFEB"). Tamoxifen administration in the chow fed to M $\phi$-TFEB mice for 2 weeks resulted in a 4.6-fold upregulation of TFEB in induced pMACs (Supplemental Figure $3, \mathrm{~A}$ and $\mathrm{B}$ ) as well as increased levels of the lysosome-associated membrane protein 1 (Lamp1), lysosomal enzyme cathepsin D, and LC3-II. TFEB activation also nonsignificantly reduced levels of p62 (Supplemental Figure 3, A and E), consistent with increased autophagic flux in the setting of probable concomitant transcriptional upregulation, given that p62 is a TFEB target. These results demonstrated that $\mathrm{Cx} 3 \mathrm{Cr} 1$ promoter-driven overexpression of TFEB in macrophages resulted in TFEB activation, which was sufficient to stimulate the autophagy-lysosome pathway.

We next tested whether Cx3Cr1-dependent TFEB overexpression attenuates myocardial IR injury and improves cardiac function after IR, in vivo. Accordingly, Cre-only control and M $\phi$-TFEB mice carrying the $\mathrm{Cx} 3 \mathrm{Cr} 1$-erCre and TFEB overexpression cassette underwent surgical implantation of the 
A
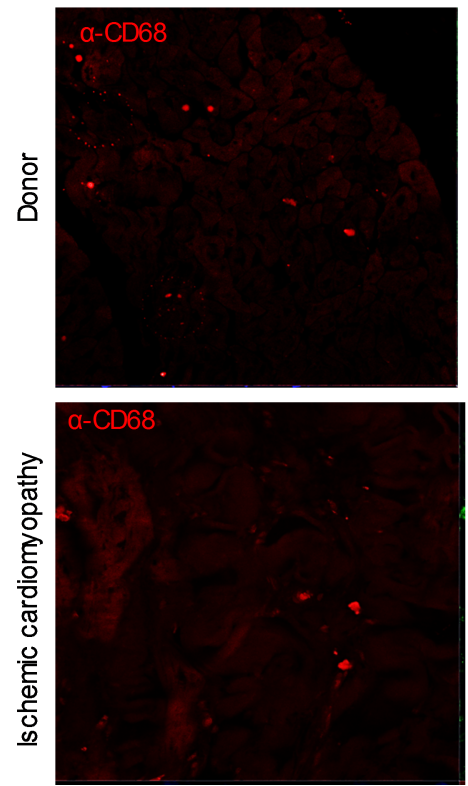
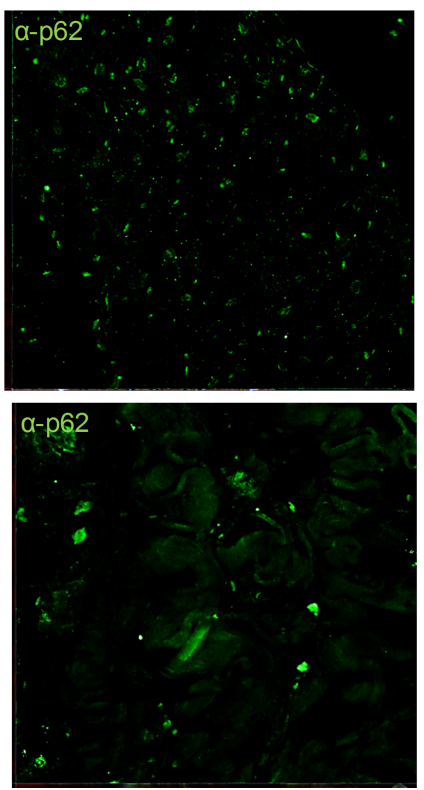
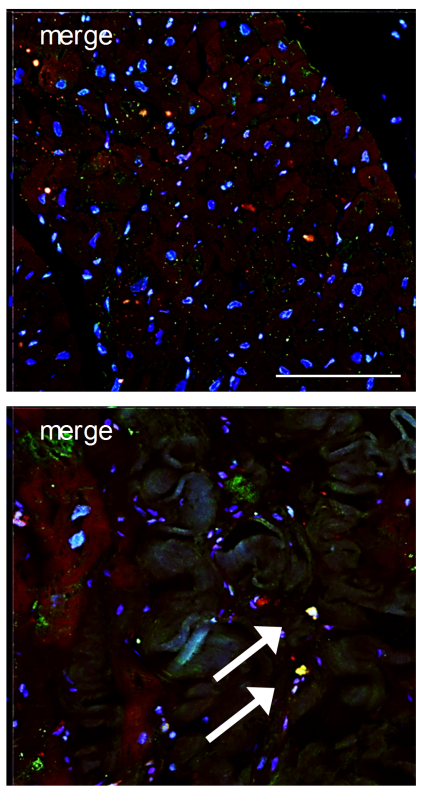

B

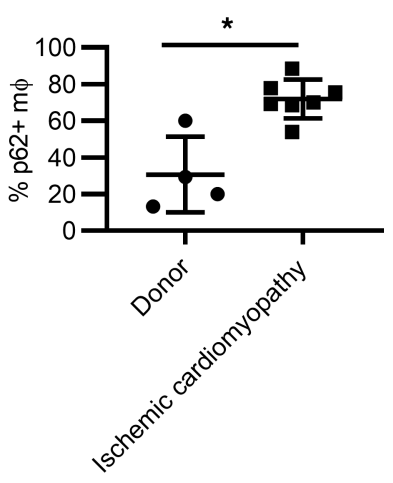

Figure 1. Autophagy is altered in human cardiac macrophages in ischemic cardiomyopathy. (A) Immunohistochemistry demonstrating anti-CD68 and anti-p62 in human hearts (donor versus ischemic cardiomyopathy). Arrows point to macrophages stained with CD68 expressing p62 by immunohistochemistry. Scale bar $=100 \mu \mathrm{m}$. (B) Quantification of staining in A. $n=4$ Donor, $n=9$ ischemic cardiomyopathy samples per group. ${ }^{*} P<0.05$ by Mann-Whitney $U$ test with individual data points, mean, and standard error shown in $\mathbf{B}$.

suture and echocardiography at day $=-14$ (Figure $3 \mathrm{~A}$ ) and were allowed to recover from surgery prior to initiation of a tamoxifen diet for 7 days. At day $=0$, mice underwent echocardiography and surgical induction of ischemia for 90 minutes, followed by reperfusion. Area-at-risk was calculated based on regional wall motion abnormalities documented during ischemia by echocardiography. Four weeks later, control animals subjected to IR injury showed adverse LV remodeling with LV dilation and decreased ejection fraction (EF) from the baseline (Figure 3, B-D) consistent with the development of cardiomyopathy after MI, mimicking what might be expected in humans with heart failure after MI (36). In contrast, M $\phi$-TFEB mice recovered their average EF to significantly higher values than in control mice, from a mean value of $26 \% \pm 1 \%$ in controls to $36 \% \pm 2 \%$ in $\mathrm{M} \phi$-TFEB (Figure $3, \mathrm{~B}$ and C). In addition, LV end-diastolic volume (EDV) was significantly smaller in M $\phi$-TFEB mice at 4 weeks after IR versus controls (Figure 3D), whereas the area-at-risk (ischemic area, Figure 3E) and the LVEF during ischemia (Figure 3C) were very similar between the 2 groups. In addition, the scar size at 4 weeks was similar between the groups (Figure 3, F and G), suggesting that the observed differences in ventricular function at 28 days were not the result of differences in the initial ischemic injury. These results were consistent with the hypothesis that $\mathrm{M} \phi$-TFEB expression is sufficient to improve ventricular function after IR injury.

$M \phi$-TFEB reduces $C C R 2^{+}$macrophages in the myocardium. To understand the role of TFEB in the inflammatory response after IR and in myocardial remodeling, we first performed immunohistochemistry of macrophages in the infarct zone and remote myocardium after closed-chest IR. Although the number of infiltrating macrophages was similar in the infarct zone of both control and M $\phi$-TFEB mice 28 days after IR injury (Figure 4, A and B), we observed a significant reduction in macrophages in remote myocardium (Figure 4, A and C) in M $\phi$-TFEB mice after IR.

Prior studies have suggested that $\mathrm{CCR} 2^{+}$macrophages are recruited into the myocardium upon injury and persist in the failing adult heart $(4,5,8,10,30,37)$ and treatment with a CCR2 inhibitor enhanced repair with preservation of resident $\mathrm{CCR} 2^{-}$subsets (38). We examined the proportion of $\mathrm{CCR} 2^{+}$macrophages in the closed-chest MI model at day 28 and observed a statistically significant reduction in both $\mathrm{CCR}^{+}$cells and the ratio of CCR2 ${ }^{+}$to $\mathrm{CCR} 2^{-}$cells in $\mathrm{M} \phi$-TFEB versus control hearts (Figure 4, D and E). These finding were consistent with the hypothesis that M $\phi$-TFEB expression influences the relative abundance of $\mathrm{CCR} 2^{+}$and $\mathrm{CCR} 2^{-}$macrophages populations after IR injury (39). To determine whether the reduction in $\mathrm{CCR} 2^{+}$macrophages may be the result of a reduction in circulating monocytes, we 
A
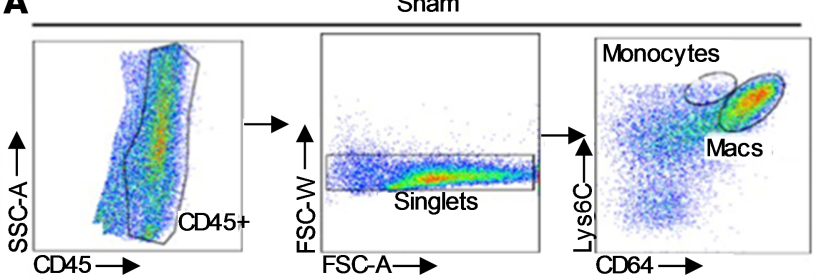

Post IR (day 4)

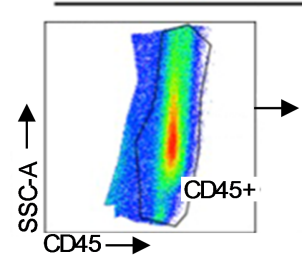

B

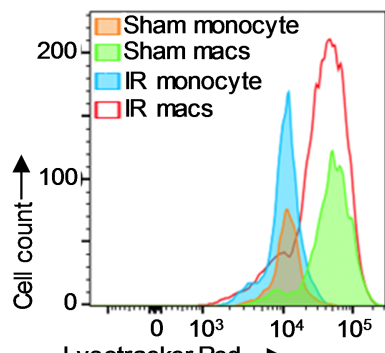

Lysotracker Red $\longrightarrow$

D

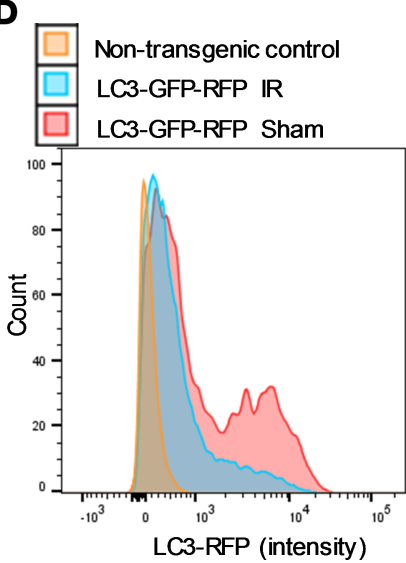

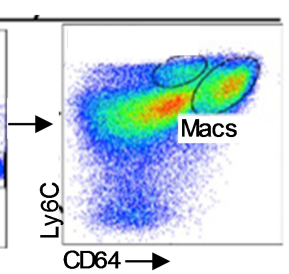

$\mathrm{FSC}-\mathrm{A} \rightarrow$

c
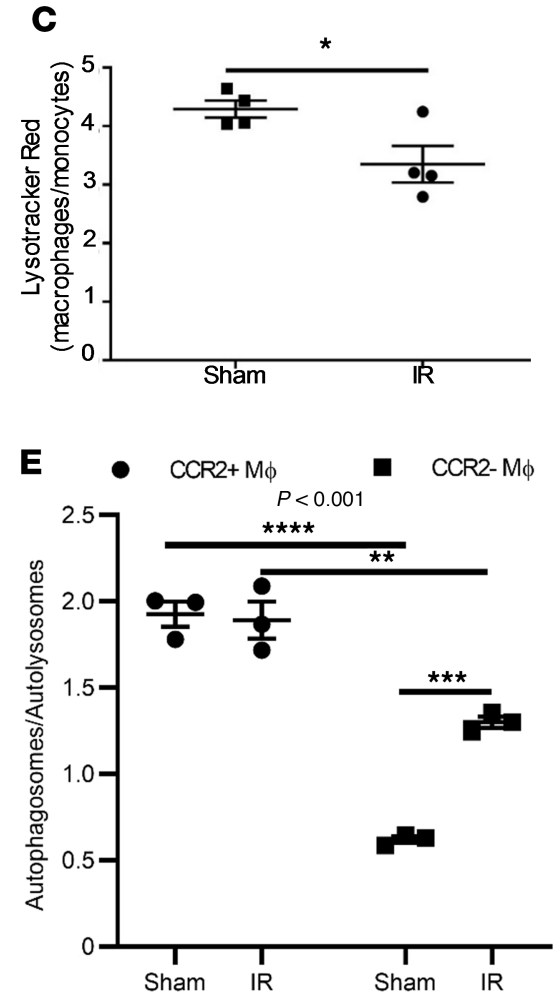

Figure 2. Autophagy and lysosomal biogenesis are perturbed after cardiac ischemia/reperfusion injury. (A) Flow-gating strategy to identify monocytes and macrophages in hearts from mice subject to sham or ischemia/ reperfusion (IR) surgeries 4 days after IR. (B) Histogram depicting LysoTracker Red staining in monocytes and macrophages from sham versus day 4 hearts after IR. (C) Quantification of LysoTracker Red mean fluorescence intensity in macrophages versus monocytes at day 4. $n=4$ /group, Student's $t$ test. (D) Representative histogram of LC3-RFP signal intensity ( $x$ axis) 7 days after closed-chest IR or sham surgery. (E) Ratio of autophagosomes/autolysosomes in CCR2+ versus CCR2- macrophages after closed-chest IR or sham surgery. $n=3$ /group, 2 -way ANOVA with multiple comparison (Tukey's $t$ test), with interaction $P$ value shown on graph. Individual data points with mean and standard error are shown on each graph. ${ }^{*} P<0.05 ;{ }^{* *} P<0.01 ;{ }^{* *} P<$ $0.001 ;{ }^{* * *} P<0.0001$.

measured circulating monocytes 7 days after MI. We observed an approximately $65 \%$ reduction in mean circulating monocytes in M $\phi$-TFEB mice that was not statistically significant $(P=0.08)$ (Supplemental Figure 4) but suggested that the reduction in CCR2 ${ }^{+}$cells observed in M $\phi$-TFEB mice may at least be partially attributable to reductions in circulating monocytes.

ATG5-dependent autophagy is dispensable for myocardial benefits of $M \phi$-TFEB to attenuate myocardial dysfunction after IR. Previously, we found that M $\phi$-TFEB attenuated atherosclerosis and this benefit was lost with concomitant ablation of ATG5, an enzymatic protein critical for initiation of autophagy (27). Based on these data, our initial hypothesis was that, akin to M $\phi$-TFEB effects in atherosclerosis, TFEB-dependent rescue was most likely to be autophagy dependent, and hence TFEB would demonstrate epistasis to ATG5. To test whether autophagy was required to mediate the beneficial effect of macrophage TFEB on myocardial function, we performed closed-chest IR injury in M $\phi$-TFEB ATG5 ${ }^{-/}$ mice (with inducible CX3CR1erCre-mediated overexpression of TFEB and excision of ATG5 in mice homozygous for floxed ATG5 alleles) versus M $\phi$-ATG5 ${ }^{-/-}$control mice. Surprisingly, our data demonstrated that M $\phi$-TFEB rescued the decline after IR in LVEF at 4 weeks even with concomitant ablation of ATG5 (Figure 5A), without significantly affecting LV dilation (Figure 5B), whereas the area-at-risk 


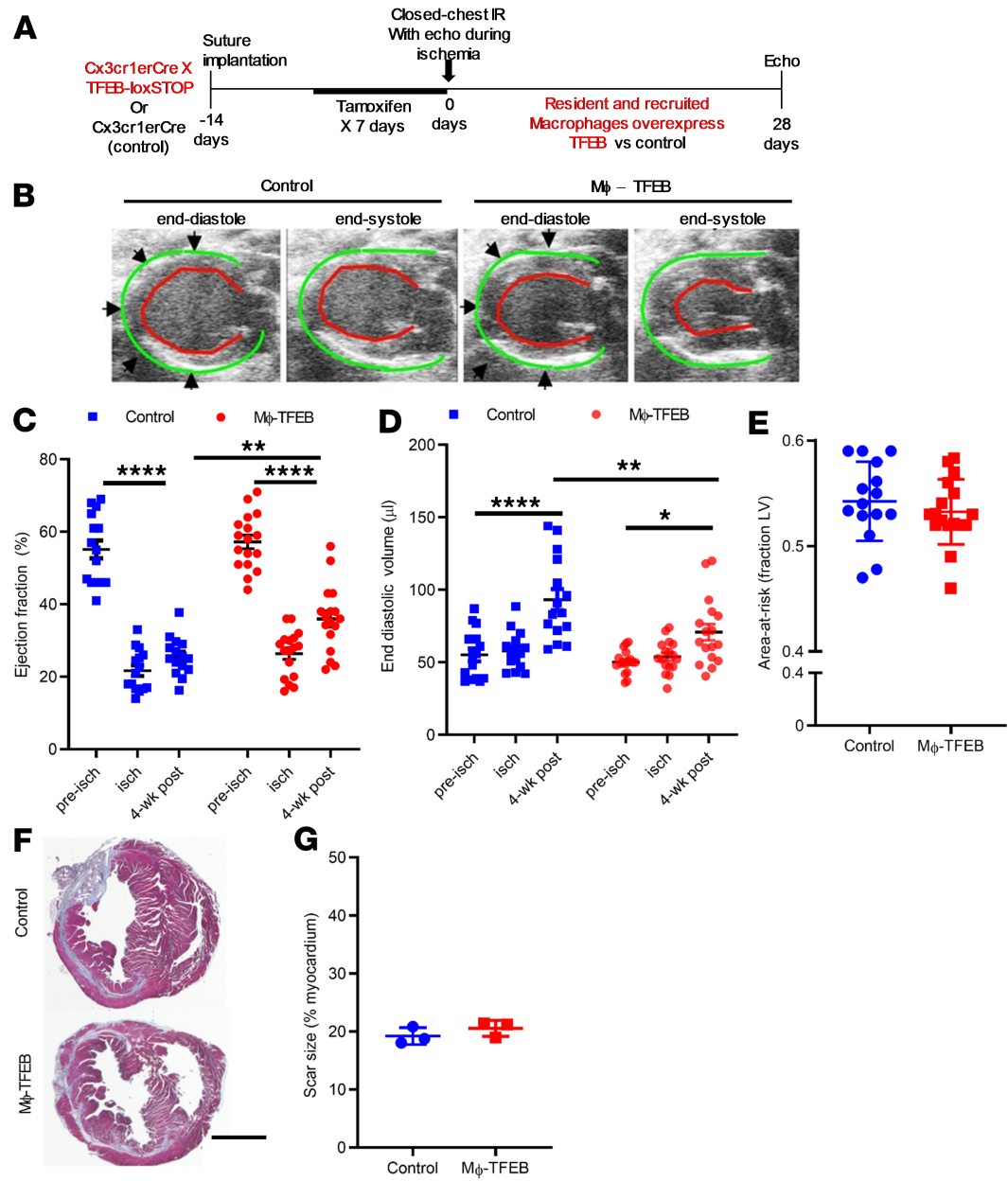

Figure 3. $M \phi$-TFEB attenuates ventricular dysfunction after ischemia/reperfusion. (A) Schematic detailing closed-chest ischemia/reperfusion (IR) protocol in control versus M $\phi$-TFEB mice. (B) Representative 2D-echocardiographic images from control versus M $\phi$-TFEB 4 weeks after IR. Green lines highlight the epicardial border; red lines demarcate the endocardium. (C) Ejection fraction (\%, $y$ axis) in control versus M $\phi$-TFEB prior to (pre-isch), during (isch), and 4 weeks after IR injury. Controls, $n=15$; M $\phi$-TFEB, $n=17$. 2-way ANOVA with multiple comparisons (Tukey's $t$ test). (D) End diastolic volume in mice modeled as in B. (E) Area-at-risk ( $y$ axis) in mice modeled as in B. Student's $t$ test. (F and $\mathbf{G}$ ) Representative Masson's trichrome stained cardiac tissue (F) from control and $\mathrm{M} \phi$-TFEB hearts 4 weeks after IR, with quantification of scar size (G) and 2-way Student's $t$ test. Scale bar $=1$ $\mathrm{mm} . n=3$ /group. Individual data points with mean and standard error are shown on each graph. ${ }^{*} P<0.05 ;{ }^{*} P<$ $0.01 ; * * * P<0.0001$

and scar size were comparable (Figure 5, C-E). These findings indicate that genetic ablation of ATG5-dependent autophagy in macrophages is dispensable for the beneficial effects of Mac-TFEB on LV dysfunction after IR.

$M \phi$-TFEB attenuates myocardial IL-1 $\beta$ protein levels after IR. We previously demonstrated that $\mathrm{M} \phi$-TFEB attenuates IL-1 $\beta$ secretion in macrophages in an autophagy-independent manner (27). Given that CCR2 ${ }^{+}$ monocytes and macrophages are a major source of IL-1 $\beta$ (11), we hypothesized that M $\phi$-TFEB may be reducing IL-1 $\beta$ levels in the myocardium. We measured IL-1 $\beta$ protein levels in 2 independent cohorts of mice at 4 days after IR (Figure 6A) and immediately after IR (Figure 6B), when IL-1B production has been observed to peak after MI (40). M $\phi$-TFEB significantly reduced IL-1 $\beta$ levels in the myocardium 1 day after IR; however, we did not observe significant changes in IL-1 $\beta$ in serum (Figure 6C), consistent with a prominent role for TFEB in myocardial macrophages. In addition, pMACs from M $\phi$-TFEB mice demonstrated significantly lower IL-1 $\beta$ mRNA abundance compared with controls in response to LPS challenge (Figure $6 \mathrm{D})$. To test the role of autophagy in TFEB-mediated IL-1 $\beta$ suppression in vitro, we performed treatment of pMACs with the specific autophagy inhibitor Spautin-1 (41). Spautin-1 treatment of control pMACs 
A

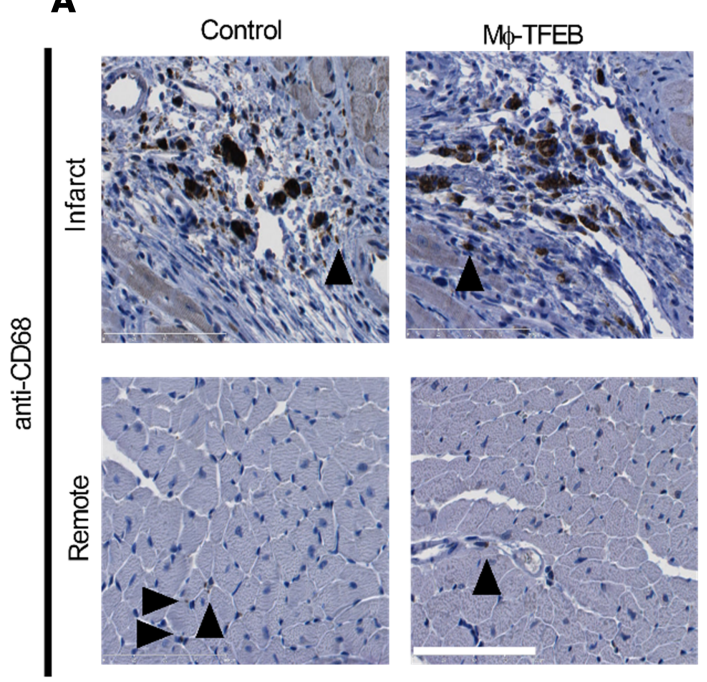

B

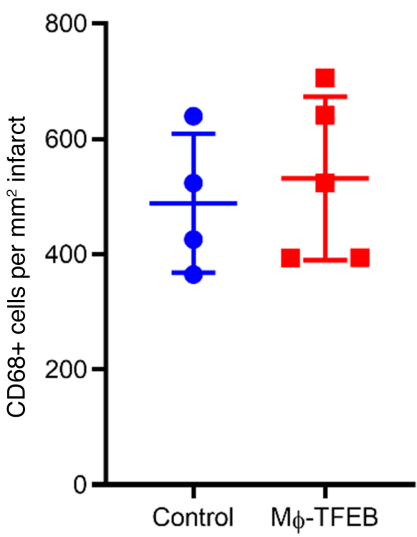

D

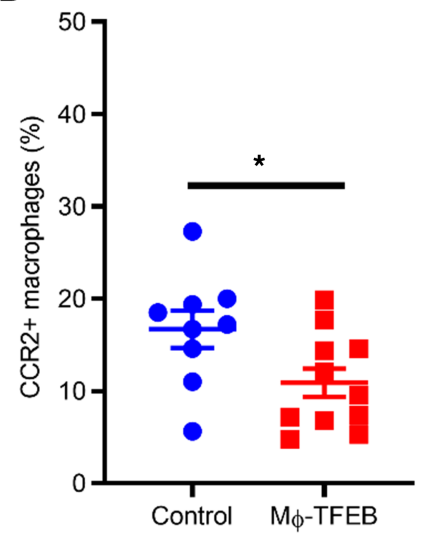

C

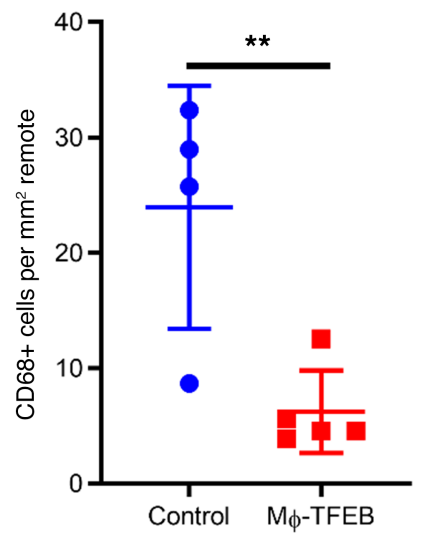

$\mathbf{E}$

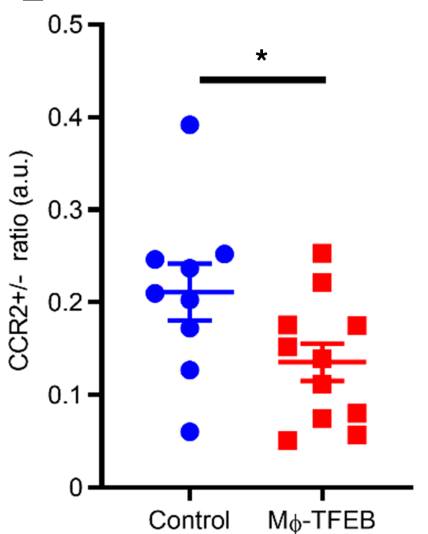

Figure 4. $M \phi$-TFEB alters inflammatory cell composition after ischemia/reperfusion in the myocardium. (A) Representative anti-CD68 stained (black arrows) image in infarcted and remote myocardium with hematoxylin counterstaining. Scale bar $=100 \mu \mathrm{m}$. (B and C) Quantification of CD68 ${ }^{+}$ cells in infarct and remote myocardium in control versus M $\phi$-TFEB mice 28 days after ischemia/reperfusion (IR). $n=4$, control; $n=5 \mathrm{M} \phi$-TFEB. (D) Percentage of CCR2 ${ }^{+}$macrophages assessed by flow cytometry 28 days after IR in control $(n=9)$ versus Mf-TFEB $(n=11)$ mice. (E) Ratio of CCR2 ${ }^{+}$. CCR2- macrophages in control $(n=9)$ versus M M-TFEB $(n=11)$ mice 28 days after IR. Individual data points with mean and standard error are shown on each graph. ${ }^{*} P<0.05 ;{ }^{*} P<0.01$ by Student's $t$ test.

resulted in robust suppression of IL-1 $\beta$ secretion after LPS/ATP stimulation, whereas concomitant TFEB overexpression and autophagic inhibition with Spautin-1 resulted in even lower secreted IL-1 $\beta$ protein levels (Figure 6E). These results are consistent with our prior findings that TFEB suppressed inflammasome activation independent of autophagy (27).

RNA-seq suggests TFEB regulates macrophage inflammatory responses via lysosomal lipolysis. To characterize the nature of TFEB-induced gene expression changes in macrophages in the myocardium after IR with an unbiased RNA-seq-based approach, we generated control and M $\phi$-TFEB mice crossed with a Rosa-TdTomato reporter (42) and fed tamoxifen for 1 week immediately followed by closed-chest IR modeling. Fluorescence-assisted cell sorting was used to separate cardiac macrophages by TdTomato labeling and CCR2 expression (Figure 7A). This strategy permitted us to identify TFEB expressing (TdTomato-labeled) macrophages stratified by their CCR2 expression pattern after IR injury (see schematic in Figure 7A). We chose to perform macrophage sorting 7 days after IR in this system because at that time point we observed both autophagic and lysosomal dysfunction in the setting of after IR (Figure 2). We subjected these 4 cell populations to RNA-seq analysis in M $\phi$-TFEB-transgenic mice $(n=4)$ and Cre-expressing (TFEB nontransgenic) control mice $(n=5)$ after IR (principle component analysis shown in Supplemental Figure 5). RNA-seq analysis uncovered 602 transcripts with greater than $1.5 \times$ fold change and a false-discovery rate of less than 0.02 that exhibited a change in differential expression secondary to TFEB overexpression (see Supplemental Table 1). Unbiased hierarchical clustering of the 602 transcripts revealed that M $\phi$-TFEB altered gene expression in both CCR2 ${ }^{+}$and CCR2- macrophages in a similar pattern (Figure 7B). Targets that demonstrated a significant increase in expression 

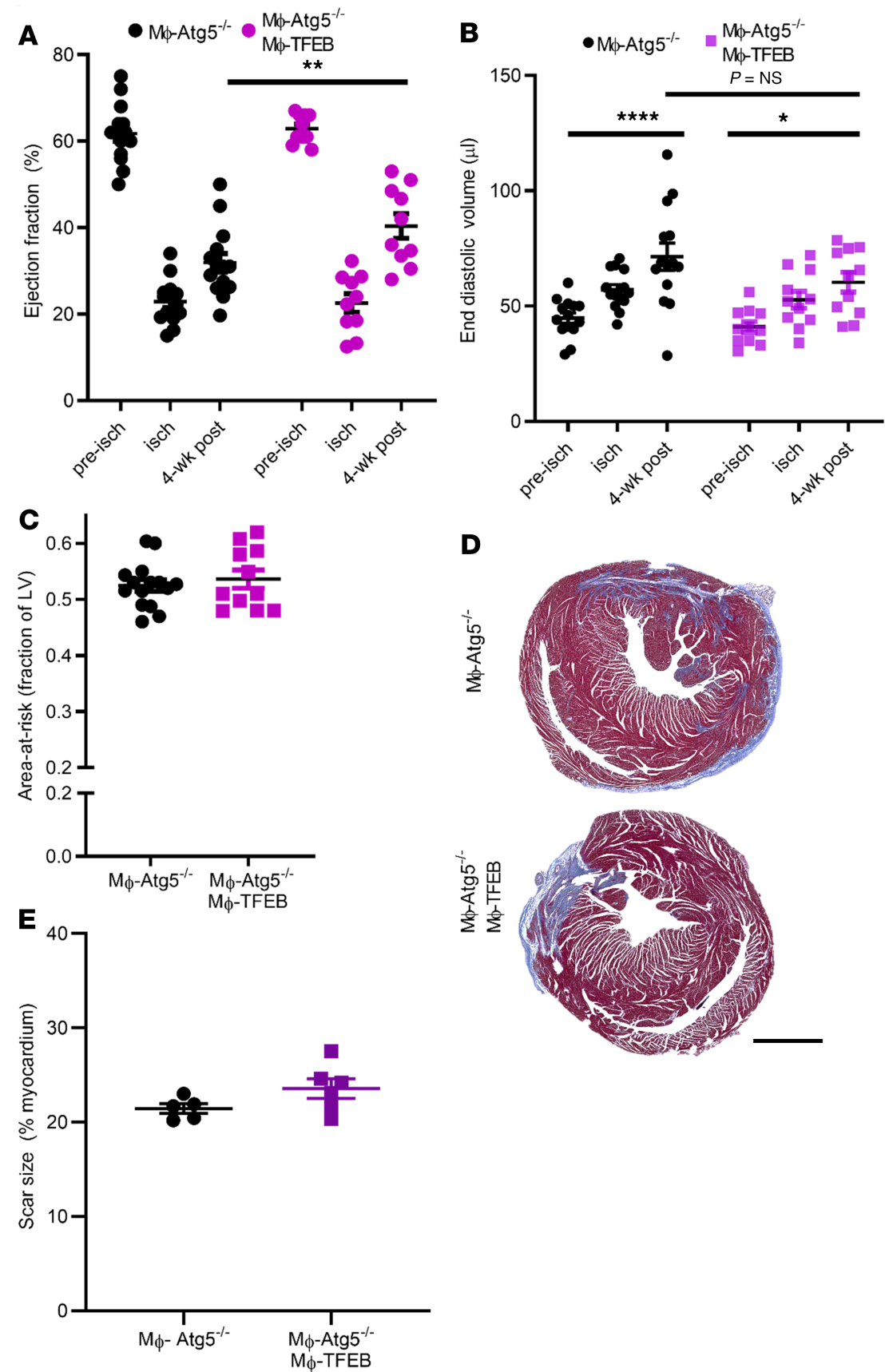

Figure 5. ATG5-depenent autophagy is not required for attenuation of ventricular dysfunction after IR by M $\phi$-TFEB (A) Ejection fraction (\%, $y$ axis) in M $\phi$-Atg $5^{-/-}$versus $\mathrm{M} \phi$-TFEB Atg $5^{-/-}$prior to (pre-isch), during (isch), and 4 weeks after ischemia/reperfusion (IR) injury. $n=15 \mathrm{M} \phi$-Atg5 $5^{-1-} ; n=11 \mathrm{M} \phi$-Atg5 $5^{-1-} \mathrm{M} \phi$-TFEB, 2-way ANOVA with multiple comparisons (Tukey's $t$ test). (B) End-diastolic volume in animals modeled as in A. (C) Area-at-risk ( $y$ axis) during ischemia from mice in A, Student's $t$ test. (D and E) Representative Masson's trichrome stained cardiac tissue (D) and quantification

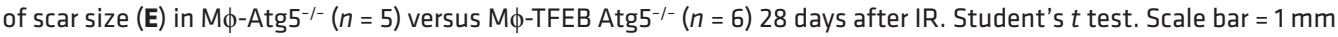
Individual data points with mean and SEM are shown on each graph. ${ }^{*} P<0.05 ;{ }^{* *} P<0.01 ;{ }^{* * *} P<0.0001$

in $\mathrm{M} \phi$-TFEB macrophages included TFEB itself (16-fold increase); in addition, we found significant upregulation of lipases, such as endothelial lipase (16-fold), LAL (1.5-fold), and the lysosomal proton pump subunit Atp6v0d2 (8-fold). Mechanistically, we were particularly interested in LAL because it has been implicated in regulation of macrophage function (43) and is required for cholesterol efflux, which can suppress NOD-like receptor family pyrin domain-containing 3 (NLRP3) inflammasome activation in atherosclerosis models $(44,45)$. In addition, we had previously demonstrated that LAL is a target of 

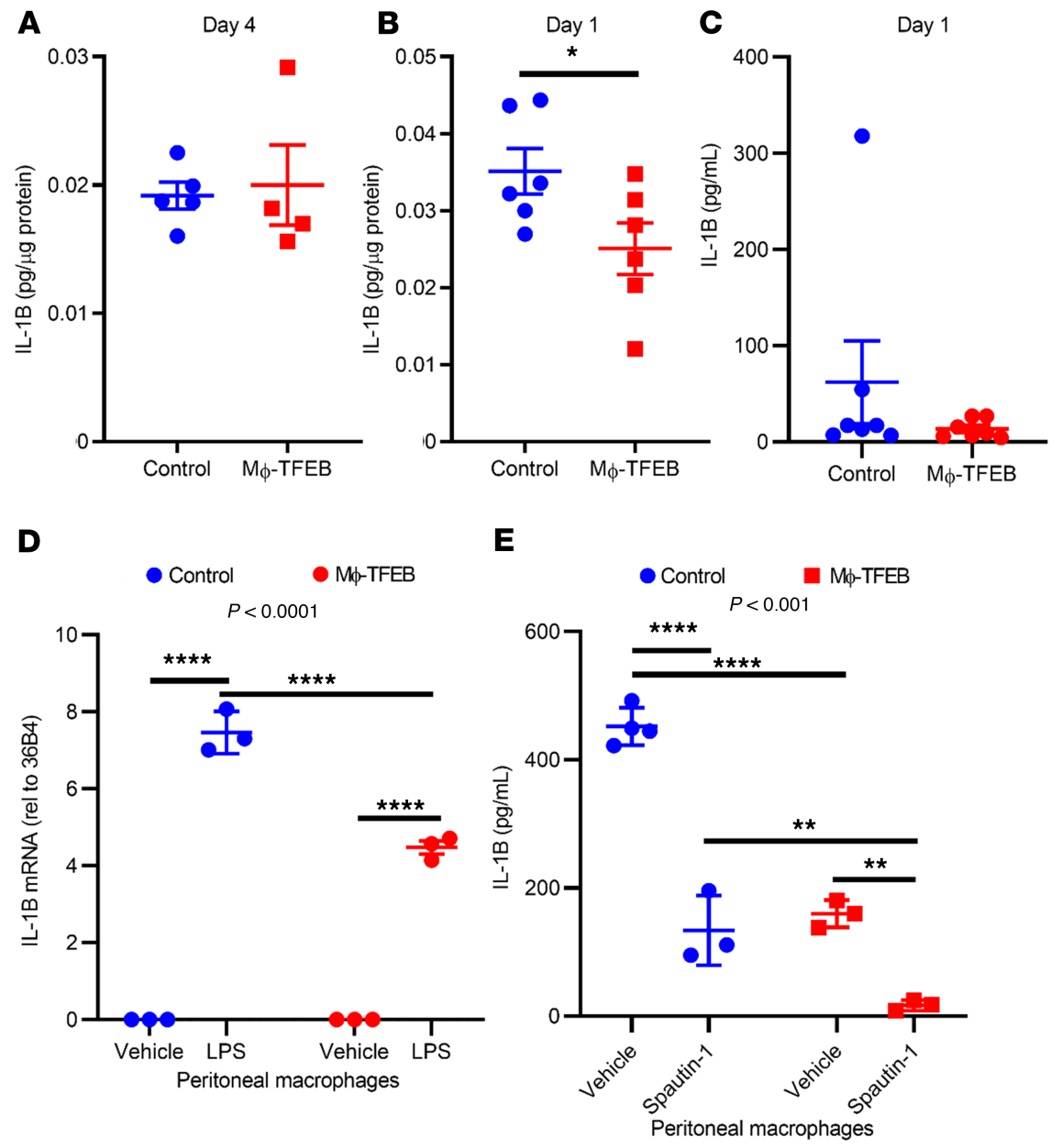

Figure 6. M $\phi$-TFEB attenuates IL-1 $\beta$ secretion independent of autophagy. (A and B) IL-1 $\beta$ myocardial protein levels at day 4 (A, $n=5$ controls; $n=4$ M $\phi$-TFEB) and at day 1 (B, $n=6$ controls; $n=6 \mathrm{M} \phi$-TFEB) after ischemia/reperfusion (IR) (C) Serum IL-1 $\beta$ levels at day 1 after IR. $n=7 /$ group, Student's $t$ test for A-C. (D) IL-1 $\beta$ mRNA from peritoneal macrophages (pMACs) stimulated with LPS for 4 hours, $n=3$ /group, 2-way ANOVA with Tukey's $t$ test for multiple comparisons; interaction $P$ value shown at top of graph. (E) IL-1 $\beta$ protein levels in supernatants from pMACs pretreated with diluent or Spautin-1 (10 $\mu \mathrm{M}$ ) for 30 minutes followed by stimulation with LPS ( 2 hours) and ATP (30 minutes). $n=3-4$ cell replicates/time point, 2-way ANOVA with Tukey's $t$ test for multiple comparisons and interaction $P$ value shown on graph. For $\mathbf{D}$ and $\mathbf{E}$ only, representative data are shown with cellular replicates from cells isolated on a single day; all peritoneal macrophage experiments were repeated at minimum 3 times on separate days; individual data points with mean and standard error are shown on each graph. ${ }^{*} P<0.05$; ${ }^{* *} P<0.01 ;{ }^{* *} P<0.001$; ${ }^{* * *} P<0.0001$.

TFEB in macrophages and is necessary for the effects of TFEB on cholesterol efflux (46). We therefore tested the role of LAL in inflammasome activation by stimulating pMACs with LPS/ATP. We found that secreted IL-1 $\beta$ protein levels were significantly reduced by M $\phi$-TFEB and that inhibition of LAL increased IL1- $\beta$ levels in both control and M $\phi$-TFEB media, suggesting that one pathway by which TFEB attenuates IL-1 $\beta$ requires intact LAL activity (Figure 7C). To test the effects of LAL inhibition in vivo on M $\phi$-TFEB-mediated suppression of remodeling after IR and ventricular dysfunction, we performed closed-chest IR in 2 cohorts of control and M $\phi$-TFEB male mice injected with either Lalistat $(20 \mathrm{mg} / \mathrm{kg}$ ) or vehicle. Whereas vehicle treatment recapitulated the effects of M $\phi$-TFEB on LV dysfunction after MI and remodeling (Figure 7, D and E), pharmacological inhibition of LAL completely abrogated these beneficial effects of macrophage TFEB activation.

\section{Discussion}

Macrophages play a central role in cardiovascular disease, including atherosclerosis and MI, and during the response to IR injury (1, 2, 4-6, 47-49). The autophagy-lysosome pathway has been implicated in the progression of atherosclerosis, and the common comorbidities of cardiovascular disease 
A

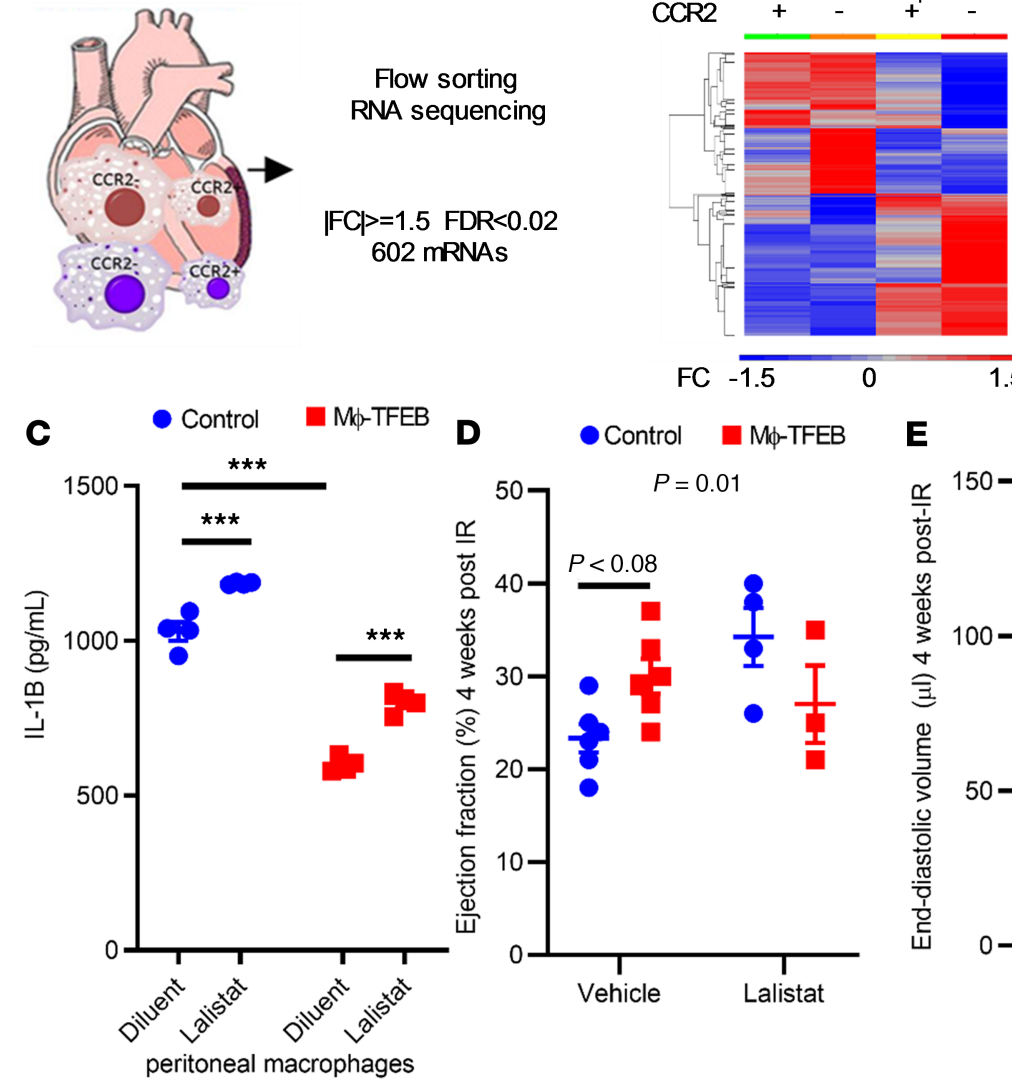

B

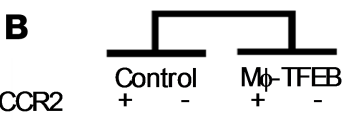

E

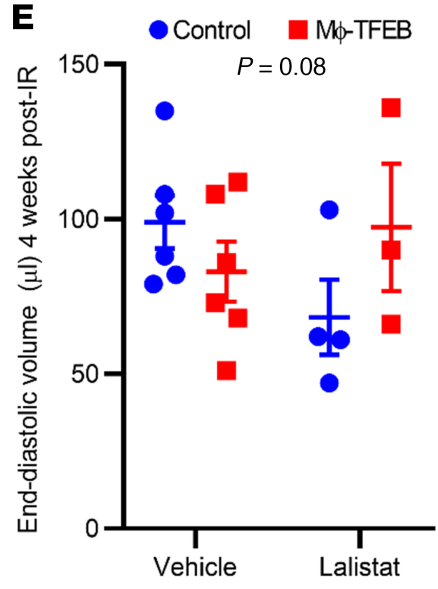

Figure 7. RNA-seq identifies lysosomal acid lipase as critical for $M \phi$-TFEB in vivo effects. (A) Diagram describing RNA-seq of flow-sorted macrophages 7 days after ischemia/reperfusion (IR). (B) Unbiased hierarchical clustering of mRNAs differentially expressed in M $\phi$-TFEB versus control cardiac macrophages. $n=4-5$ mice/group. (C) Lysosomal acid lipase (LAL) inhibition increases IL-1 $\beta$ secretion in peritoneal macrophages (pMACs) stimulated with LPS and ATP. $n=3$ /cellular replicates from cells isolated on a single day; all peritoneal macrophage experiments were repeated at minimum 3 times on separate days. 2-way ANOVA with multiple testing correction (Tukey's $t$ test). ( $\mathbf{D}$ and $\mathbf{E}$ ) Ejection fraction and end-diastolic volume in control and M $\phi$-TFEB mice treated with vehicle ( $n$ $=6$ ) or Lalistat $(n=4), 2$-way ANOVA with multiple comparison correction (Sidak test, with interaction $P$ value shown on graph). Individual data points with mean and standard error are shown on each graph. ${ }^{* *} P<0.001$.

may cause impairment in autophagy (31). Here we demonstrated that autophagic insufficiency occurs in cardiac macrophages in the setting of ischemic cardiomyopathy, which may be the result of a combination of risk factors, including dyslipidemia and diabetes mellitus. However, we showed that IR injury and hypoxia/reoxygenation were sufficient to trigger autophagosome accumulation, which indicated lysosomal insufficiency and/or dysfunction. We have previously shown that TFEB, a master regulator of autophagy and lysosomal biogenesis $(23,24)$, can attenuate lipotoxic stress in macrophages $(50)$, attenuate IL-1B secretion, and reduce atherosclerotic progression (27). We have shown that M $\phi$-TFEB attenuates myocardial dysfunction after IR, improves LVEF, and reduces IL-1 $\beta$ in the LV and in pMACs. In contrast to the observed benefits of TFEB in the setting of atherosclerosis, which depend on intact autophagy, loss of ATG5, a key protein involved in phagophore membrane extension, did not circumvent the benefits of TFEB on LV dysfunction after IR. To understand the in vivo mechanism by which M $\phi$-TFEB attenuates LV dysfunction after IR and remodeling, we performed RNA-seq of flow-sorted macrophages and identified LAL, a known TFEB target. We showed that inhibition of LAL with Lalistat was sufficient to abrogate the beneficial effects of TFEB in vivo. In summary, we proposed that TFEB-mediated attenuation of myocardial dysfunction was likely attributable to its role mediating lysosomal lipolysis via LAL and independent of ATG-5-dependent autophagy. Our findings implicated a central role for lysosome dysfunction and not primarily autophagy impairment (which is 
a consequence of lysosome dysfunction) in provoking macrophage-driven inflammation in ischemic cardiomyopathy.

Our findings were also consistent with the work of others demonstrating the complex role of autophagy in inflammasome activation, which has garnered markedly increased attention since the publication of the CANTOS trial (12). Full inflammasome activation requires 2 independent steps. "Signal one," or priming, occurs when NF- $\mathrm{KB}$ activation is induced downstream of toll-like receptor 4 or TNF receptor signaling leading to transcriptional activation of NLRP3, pro-IL-1 $\beta$, and pro-IL-18. Subsequently, multiple stimuli can act as a "signal two" to induce NLRP3 activation, robust IL-1 $\beta$ secretion with cleavage, and activation of caspase-1. Of note, autophagy has been implicated in IL-1 $\beta$ suppression. ATG5 ablation, for example, increases IL-1 $\beta$ production when macrophages are treated with LPS alone (without a second signal for bona fide inflammasome assembly, IL-1 $\beta$ processing, and secretion) (51). In addition, autophagy targets NLRP3 for lysosomal degradation and directly regulates inflammasome activation via ROS (52). An important caveat of these studies is that they mostly used LPS-treated macrophages, in contradistinction to full NLRP3 activation, which requires 2 signals. Our results were consistent with these findings, insofar as when macrophages were treated with LPS and palmitate, autophagy ablation also resulted in reduced IL-1 $\beta$ secretion (53).

Several reports have highlighted an additional role for autophagy in IL-1 $\beta$ secretion. Specifically, an unconventional autophagy-mediated secretory pathway regulates IL-1 $\beta$ secretion in both neutrophils and macrophages $(15,19,54)$, where ATG5 was demonstrated to have a critical role. In the setting of myocardial IR injury, macrophages encounter hypoxia/reoxygenation that we showed can impair autophagy, concomitant with the release of damage-associated molecular patterns that can activate NLRP3 through direct and indirect mechanisms. In addition, we showed that inhibition of lysosomal lipolysis with Lalistat also increases IL-1 $\beta$ secretion. Therefore, during IR injury, macrophage lysosomes serve complex roles in inflammasome suppression via LAL but also in Atg5-dependent IL-1 $\beta$ secretion.

After myocardial injury, the bone marrow and spleen release CCR2 ${ }^{+}$cells that home to the myocardium and differentiate into $\mathrm{CCR} 2^{+}$macrophages. CCR2 ${ }^{+}$cells are a major source of proinflammatory cytokine production, including IL-1 $\beta$ (11). Macrophage TFEB overexpression resulted in a reduction in the percentage of $\mathrm{CCR}_{2}{ }^{+}$macrophages in the myocardium, consistent with TFEB-mediated reductions in IL-1 $\beta$. Accordingly, our data suggested that M $\phi$-TFEB induction was primarily effective in abrogating remodeling after IR via its effects in recruited and not resident macrophages. However, just as the net effects of autophagy on IL-1 $\beta$ are complex, TFEB itself has dual roles in regulation of inflammation. Studies by the Puertollano laboratory have highlighted the role of TFEB and its family member TFE3 as stress sensors that are required for the inflammatory response and IL-1 $\beta$ production (28). Both our previous report (27) and current studies demonstrated that TFEB overexpression was also sufficient to modulate the inflammatory response. It is very possible that both reductions and increases in TFEB activity can suppress IL-1 $\beta$ production. Whether or not overexpression of TFE3 is sufficient to attenuate IL-1 $\beta$ production is unknown. In aggregate, it is best to consider TFEB actions as immunomodulatory rather than either a driver or suppressor of IL-1 $\beta$.

To understand the mechanisms by which TFEB may attenuate myocardial dysfunction after IR injury, we performed RNA-seq on flow-sorted $\mathrm{CCR} 2^{+}$and $\mathrm{CCR} 2^{-}$macrophages isolated from the myocardium 7 days after IR injury. Broadly, these studies suggested that TFEB might be altering gene expression in $\mathrm{CCR} 2^{+}$and $\mathrm{CCR} 2^{-}$macrophages in a similar fashion. Consistent with prior studies that demonstrated the ability of TFEB to induce the expression of multiple genes involved in lipid catabolism (55), we found that TFEB induced the expression of several lipases in macrophages in vivo. In particular, our data showed that TFEB upregulates LAL expression in macrophages, consistent with a prior report of lysosomal lipase induction by HLH-30 (TFEB ortholog) in C. elegans $(56,57)$. Interestingly, we implicated LAL in inflammasome suppression and suggest a critical role for LAL in TFEB-dependent attenuation of injury after IR, in vivo. LAL inhibition increases IL-1 $\beta$ secretion in response to LPS+ATP, suggesting that increased LAL may at least be partially responsible for the effects of TFEB on IL-1 $\beta$. Identifying the precise mechanisms by which LAL may reduce IL-1 $\beta$ is of great translational interest, especially given the potential relevance of IL-1 $\beta$ secretion to human disease; clinical trials of IL-1 $\beta$ antagonists to treat cardiac dysfunction after MI are currently underway. Our studies were also consistent with the recent identification of 25-hydroxycholesterol, a product of LAL enzyme activity, as a critical target for the suppression of IL-1 $\beta$ secretion (58).

Our work should be understood in the context of its strengths and limitations. First, our human samples were from patients with chronic ischemic heart failure, whereas our animal and cell work has 
been performed at acute time points. Given that other studies have demonstrated autophagic impairment in macrophages in patients with atherosclerosis and diabetes mellitus, our observation of p62 accumulation in human cardiac macrophages was unsurprising. Herein, our mechanistic observation was that both IR injury and hypoxia/reoxygenation were sufficient to provoke autophagic impairment. Second, whereas we found that the effects of TFEB were independent of ATG5, we cannot rule out ATG5-independent autophagy as potentially being required for some effects of macrophage TFEB. Although our overall results were consistent with LAL activity being (a) involved in inflammasome suppression in vitro and (b) required for TFEB-mediated effects on remodeling after IR, it was also clear that there were other effects of TFEB on macrophages in vivo, and the effects of TFEB were unlikely to be fully explained by a single mechanism. Future studies will be designed to directly test the role of macrophage LAL in the myocardial response to injury.

In summary, our work suggests that macrophage TFEB activation is an effective strategy to attenuate myocardial dysfunction after IR and reduce IL1- $\beta$ secretion independent of ATG5-dependent autophagy. We showed that TFEB's effects are partially dependent on lysosomal acid lipase, highlighting lysosomal function, rather than autophagy per se, as a key mechanism of action of TFEB.

\section{Methods}

Samples and reagents. Transgenic mice expressing LC3 conjugated with green and red fluorescent protein (RFP-EGFP-LC3) under control CAG promoter were purchased from Jackson Laboratory (strain 027139) (35) and were bred in-house. Transgenic mice containing a Lox-STOP cassette (CAG-TFEBLoxP-STOP) (23) were crossed with $\mathrm{Cx} 3 \mathrm{Cr} 1$-er-Cre mice that were purchased from Jackson Laboratory (strain 021160) and bred in-house. LysM-Cre (used for pMAC studies) and $A T G 5^{\mathrm{f} / \mathrm{fl}}$ mice were previously described (27), as were td-tomato reporter mice (ROSA26-CAGpromoter-lox-STOP-lox-dTomato (42) (JAX stock 007909). Tamoxifen diet (500 mg/kg) was purchased from Envigo (formerly Harlan Teklad, TD 130857). LysoTracker Red DND-99 (Thermo Fisher Scientific) was used at a final concentration of 5 nM. CD45-PerCP/Cy5.5 (30-F11), CD64-APC (X54-5/7.1), CCR2-BV421 (SA203G11), MHCII-APC/ Cy7 (M5/114.15.2), Ly6C-BV510 and FITC (HK1.4), CD45.1-PE (A20) were purchased (Biolegend) and used in multichannel flow as described below. CD68 antibody clone ab125212 (Abcam) was used for immunohistochemistry as was p62/Sqstm1 GP62-C (Progen) and for immunoblotting: TFEB clone A303-673A (Bethyl Labs), p62 ab56415 (Abcam), LC3-II ab48394 (Abcam), LAMP1 sc019992 (Santa Cruz), Cathepsin D (a gift from Stuart Kornfeld, Washington University, St. Louis, Missouri, USA), and $\beta$-actin A2066 (Sigma-Aldrich). Ultrapure E. coli lipopolysaccharide (InVivoGen), ATP (Sigma-Aldrich), bafilomycin A (Sigma-Aldrich), and the autophagy inhibitor Spautin-1(41) (Sigma-Aldrich) were purchased and employed in in vitro studies with pMACs. Lalistat was provided by the University of Notre Dame, (South Bend, Indiana, USA; ref. 59). Fixed, deidentified human heart tissue obtained from Kenneth Margulies at University of Pennsylvania. Hearts were categorized as either nonfailing (no history of heart failure, obtained from nonfailing brain-dead donors) or ischemic cardiomyopathy (obtained at time of orthotopic heart transplantation). After in situ cold cardioplegia, all hearts were placed on wet ice at $4^{\circ} \mathrm{C}$ in Krebs-Henseleit buffer. Transmural LV samples were obtained from the LV free wall with epicardial fat excluded, and samples were then placed in $4 \%$ paraformaldehyde for fixation.

Closed-chest I/R surgery and echocardiography. Methods for closed-chest IR have been previously described (60). Briefly, age- and sex-matched mice were anesthetized with ketamine/xylazine (100 mg/kg and $10 \mathrm{mg} /$ $\mathrm{kg}$, i.p.), prepped for surgery, and ventilated. A left mini-thoracotomy was performed with pericardial dissection, followed by passage of an 8-0 polypropylene suture with a U-shaped tapered needle under the proximal $\mathrm{LAD}$ artery. The needle was cut from the suture and the 2 ends of the 8-0 suture were threaded through a 0.5-mm piece of PE-10 tubing that was previously gas sterilized. The suture and occluder formed a loose snare around the LAD artery. Each end of the suture was then threaded through a size 3 Kalt needle and exteriorized through each side of the chest wall and the thoracotomy was then closed. The ends of the exteriorized 8-0 suture were tucked under the skin and the skin closed. The mouse was removed from the ventilator and kept warm. Mice were then allowed adequate recovery time, during which they were fed the tamoxifen diet (depending on the experiment); subsequently, mice were reanesthetized but not ventilated, and only the skin above the chest wall was reopened. Mice were taped to an ECG board (Indus Instruments) to observe ST segment changes during IR. The suture ends were pulled apart gently until ST segment elevation appeared on the ECG indicating LAD artery occlusion. The sutures were then secured in this position and ischemia 
was continued for 90 minutes. To induce reperfusion, the sutures were cut close to the chest wall releasing the tension, and reperfusion was confirmed with resolution of ST segment elevation. Mice also underwent echocardiography during IR to assess area-at-risk and 4 weeks after IR (using $100 \mathrm{mg} / \mathrm{kg}$ i.p. tribromoethanol anesthetic). Echocardiography was performed on a Vevo 2100 (Visualsonics) machine equipped with a 30 $\mathrm{MHz}$ linear-array transducer as previously described $(61,62)$. Cardiac images were obtained by a handheld technique. EF and volume calculations were performed by a blinded echocardiographer. In experiments to test the role of LAL, vehicle $(0.5 \%$ methylcellulose $0.1 \%$ Tween 20$)$ or Lalistat $(20 \mathrm{mg} / \mathrm{kg}$ ) was injected subcutaneously, as previously described (43), 1 day prior and 1 day after IR surgery.

Flow cytometry, flow sorting, and RNA-seq. To prepare single cell suspension, hearts were perfused with saline, finely minced, and were digested in DMEM with collagenase $1(450 \mathrm{U} / \mathrm{mL})$, hyaluronidase (60 U/ $\mathrm{mL})$ and DNase $\mathrm{I}(60 \mathrm{U} / \mathrm{mL})$ for 1 hour at $37^{\circ} \mathrm{C}$. After 1 hour of digestion, enzymes samples were deactivated by washing in HBSS supplemented with $2 \%$ FBS and $0.2 \%$ BSA. Samples were filtered through $40 \mu \mathrm{M}$ cell strainers, washed in HBSS, and resuspended in $100 \mu \mathrm{L}$ of FACS buffer (DPBS with 2\% FBS and $2 \mathrm{mM}$ EDTA). Cells were then stained with monoclonal antibodies for 30 minutes at $4^{\circ} \mathrm{C}$. Samples were washed twice and final resuspension was made in $300 \mu \mathrm{L}$ of FACS buffer. CD $45^{+}$gate was used for all immune cells. Neutrophils were gated as $\mathrm{Ly} 6 \mathrm{G}^{+} \mathrm{CD} 64$. Monocytes were gated as $\mathrm{CD} 64^{+} \mathrm{Ly} 6 \mathrm{C}^{\mathrm{hi}}$. Macrophages were gated as $\mathrm{Ly} 6 \mathrm{G}^{-} \mathrm{CD} 4^{\text {hi }}{ }^{\mathrm{Ly}} 6 \mathrm{C}^{\mathrm{lo}}$. Flow cytometric analysis was performed on BD LSRII platform. Sorting of CCR2 ${ }^{+}$ and CCR2 ${ }^{-}$macrophages was performed on BD FACS ARIA III platform with $85 \mu \mathrm{M}$ nozzle and flow rate set to $1 \mu \mathrm{L} /$ minute. Sample collection holder was set at $4^{\circ} \mathrm{C}$. Cells were sorted for each population directly into the $200 \mu \mathrm{L}$ of lysis buffer (Qiagen) containing 1\% $\beta$-mercaptoethanol. RNA extraction was performed using RNeasy Micro kit (Qiagen). Additional methods describing RNA-seq are included in Supplemental methods. RNA-seq data files are available in GEO under accession number GSE118463.

Immunohistochemistry. Slides of paraffin-embedded human cardiac tissue were obtained from donors rejected for transplantation or patients with ischemic cardiomyopathy at the time of surgery. Immunohistochemistry was performed as previously described (11). The high-resolution images of Masson's trichrome-stained tissue slices were acquired using a digital whole slide scanner (Nanozoomer 2-HT) using a $20 \times / 0.75$ lens (Olympus). The images were then quantified using the image analysis module of a digital pathology software Visiomorph (VisioPharm).

Monocyte number determination. Whole blood $(20 \mu \mathrm{L})$ was loaded into Hemavet 950 FS (Erba Diagnostics).

Cell culture and hypoxia/reoxygenation experiments. pMACs were isolated from C57/BL6 mice or the relevant transgenic mice 4 days after injection of $3.85 \%$ thioglycollate and plated at a density of $1 \times 10^{6}$ cells/ $\mathrm{mL}$ in DMEM containing 10\% inactivated fetal serum, $1 \%$ sodium pyruvate, $1 \mathrm{mM} \mathrm{L}$-glutamate, $50 \mathrm{U} / \mathrm{mL}$ penicillin $\mathrm{G}$ sodium, and $50 \mathrm{U} / \mathrm{mL}$ streptomycin sulfate. On the day after isolation, cells were washed and then stimulated with $500 \mathrm{ng} / \mathrm{mL}$ lipopolysaccharide for 2 hours, prior to addition of ATP (4 mM) for 30 minutes. For hypoxia/reoxygenation modeling, macrophages were incubated in an oxygen control cabinet (Coy Laboratories), which was housed within an incubator equipped with an oxygen sensor. Cells were treated with $1 \%$ oxygen (maintained by infusing 95\% nitrogen and $5 \% \mathrm{CO}_{2}$ ) for 16 hours, followed by 8 hours of reoxygenation. In the final 2 hours, cells were also treated with either vehicle of $200 \mathrm{nM}$ bafilomycin A to inhibit lysosomal acidification prior to harvesting for immunoblotting.

Immunoblotting. After lysates were prepared from pMACs, Western blotting was performed as previously described (63).

$I L-1 \beta$ ELISA. IL-1 $\beta$ ELISA was performed on precoated plates (R\&D Systems) as previously described (64).

Quantitative PCR analyses. After RNA isolation from pMACs using the RNeasy Mini kit (Qiagen), cDNA was prepared using the iScript cDNA synthesis kit (Bio-Rad). Transcript levels of IL-1 $\beta$ and 36B4 were determined by qPCR performed on the QuantStudio 3 (Applied Biosystems) using the following primer sets: IL-1 $\beta$ (forward, 59-AAGGAGAACCAAGCAACGACAAAA-39; reverse, 59-TGGGGAACTCTGCAGACTCAAACT-39); 36B4 (forward, 59-ATCCCTGACGCACCGTGA-39).

Statistical analysis. Statistics were performed in Prism Version 8.0.2 (GraphPad Software Inc.). Statistical significance of differences was calculated via unpaired 2-tailed Student's $t$ test for 2 group comparisons or 2-way ANOVA to determine mean differences between groups split by 2 independent variables (in IR experiments) followed by either Tukey's or Sidak's adjustment for multiple comparisons. Experiments in pMACs were analyzed with 2-way ANOVA followed by Tukey's test for multiple comparisons. Autophagic flux experiments were analyzed by paired 2-tailed Student's $t$ test. Graphs containing error bars show means \pm SEM with a $P$ value $<0.05$ considered statistically significant. 
Study approval. All animal studies were approved by the Animal Studies Committee at Washington University School of Medicine and the IACUC committee at the John Cochran VA Medical Center (St. Louis, Missouri, USA). Studies on human tissue were performed under an exemption by the IRB at Washington University School of Medicine because only deidentified human samples were used.

\section{Author contributions}

AJ wrote the manuscript, participated in conception and design of the experiments, and performed experiments. GB, AP, SJM, LF, HE, AK, CJW, KH, and QX performed experiments and critically reviewed the manuscript. JML critically reviewed the manuscript. AB provided a critical reagent and critically reviewed the manuscript. SJM analyzed bioinformatics dataset and critically reviewed the manuscript. BR, JDS, and KJL critically reviewed the manuscript and provided key scientific input. AD conceived of the experiments and wrote the manuscript.

\section{Acknowledgments}

This study was supported by grants from the Department of Veterans Affairs (I01BX004235) and the NIH (HL107594) to AD. AJ was supported by 5-T32-HL007081 and K08-HL138262 from the NHLBI and by the Diabetes Research Center at Washington University in St. Louis of the NIH under award number P30DK020579. We also acknowledge Kenneth Margulies (University of Pennsylvania for providing human cardiac tissue), support from the NIH Shared Instrumentation Grant (S10 RR027552) for support through the Hope Center Neuroimaging Core, and support from the Advanced Imaging and Tissue Analysis Core of the Digestive Disease Research Core Center (DDRCC NIH P30DK052574) at Washington University School of Medicine.

Address correspondence to Abhinav Diwan, 600 South Euclid, Box 8086, Saint Louis, Missouri 63110, USA. Phone: 314.747.3457; Email: adiwan@wustl.edu.

1. Honold L, Nahrendorf M. Resident and monocyte-derived macrophages in cardiovascular disease. Circ Res. 2018;122(1):113-127. 2. Epelman S, Liu PP, Mann DL. Role of innate and adaptive immune mechanisms in cardiac injury and repair. Nat Rev Immunol. 2015;15(2):117-129.

3. Libby P, Hansson GK. Inflammation and immunity in diseases of the arterial tree: players and layers. Circ Res. 2015;116(2):307-311

4. Frantz S, Nahrendorf M. Cardiac macrophages and their role in ischaemic heart disease. Cardiovasc Res. 2014;102(2):240-248

5. Dutta P, Nahrendorf M. Monocytes in myocardial infarction. Arterioscler Thromb Vasc Biol. 2015;35(5):1066-1070.

6. Lavine KJ, et al. The macrophage in cardiac homeostasis and disease: JACC macrophage in CVD series (Part 4). J Am Coll Cardiol. 2018;72(18):2213-2230.

7. Wan E, et al. Enhanced efferocytosis of apoptotic cardiomyocytes through myeloid-epithelial-reproductive tyrosine kinase links acute inflammation resolution to cardiac repair after infarction. Circ Res. 2013;113(8):1004-1012.

8. Dutta P, et al. Myocardial infarction activates CCR2(+) hematopoietic stem and progenitor cells. Cell Stem Cell. 2015;16(5):477-487.

9. Bajpai G, et al. Tissue Resident CCR2 $2^{-}$and CCR $2^{+}$cardiac macrophages differentially orchestrate monocyte recruitment and fate specification following myocardial injury. Circ Res. 2019;124(2):263-278.

10. Leuschner F, et al. Rapid monocyte kinetics in acute myocardial infarction are sustained by extramedullary monocytopoiesis. J Exp Med. 2012;209(1):123-137.

11. Bajpai G, et al. The human heart contains distinct macrophage subsets with divergent origins and functions. Nat Med. 2018;24(8):1234-1245.

12. Ridker PM, et al. Antiinflammatory therapy with canakinumab for atherosclerotic disease. N Engl J Med. 2017;377(12):1119-1131.

13. Harouki N, et al. The IL-1 $\beta$ antibody gevokizumab limits cardiac remodeling and coronary dysfunction in rats with heart failure. JACC Basic Transl Sci. 2017;2(4):418-430.

14. Abbate A, et al. Interleukin-1 blockade with anakinra to prevent adverse cardiac remodeling after acute myocardial infarction (Virginia Commonwealth University Anakinra Remodeling Trial [VCU-ART] Pilot study). Am J Cardiol. 2010;105(10):1371-1377.e1.

15. Harris J, Lang T, Thomas JPW, Sukkar MB, Nabar NR, Kehrl JH. Autophagy and inflammasomes. Mol Immunol. 2017;86:10-15

16. Saitoh T, et al. Loss of the autophagy protein Atg16L1 enhances endotoxin-induced IL-1 $\beta$ production. Nature. 2008;456(7219):264-268.

17. Nakahira K, et al. Autophagy proteins regulate innate immune responses by inhibiting the release of mitochondrial DNA mediated by the NALP3 inflammasome. Nat Immunol. 2011;12(3):222-230.

18. Zhou R, Yazdi AS, Menu P, Tschopp J. A role for mitochondria in NLRP3 inflammasome activation. Nature. 2011;469(7329):221-225.

19. Iula L, et al. Autophagy mediates interleukin-1 $\beta$ secretion in human neutrophils. Front Immunol. 2018;9:269.

20. Zhang M, Kenny SJ, Ge L, Xu K, Schekman R. Translocation of interleukin-1 $\beta$ into a vesicle intermediate in autophagy-mediated secretion. Elife. 2015;4:e11205.

21. Hariharan N, Zhai P, Sadoshima J. Oxidative stress stimulates autophagic flux during ischemia/reperfusion. Antioxid Redox Sig- 
nal. 2011;14(11):2179-2190.

22. Ma X, et al. Regulation of the transcription factor EB-PGC1 $\alpha$ axis by beclin-1 controls mitochondrial quality and cardiomyocyte death under stress. Mol Cell Biol. 2015;35(6):956-976.

23. Settembre C, et al. TFEB links autophagy to lysosomal biogenesis. Science. 2011;332(6036):1429-1433.

24. Sardiello M, et al. A gene network regulating lysosomal biogenesis and function. Science. 2009;325(5939):473-477.

25. Ma X, et al. Impaired autophagosome clearance contributes to cardiomyocyte death in ischemia/reperfusion injury. Circulation. 2012;125(25):3170-3181.

26. Godar RJ, et al. Repetitive stimulation of autophagy-lysosome machinery by intermittent fasting preconditions the myocardium to ischemia/reperfusion injury. Autophagy. 2015;11(9):1537-1560.

27. Sergin I, et al. Exploiting macrophage autophagy-lysosomal biogenesis as a therapy for atherosclerosis. Nat Commun. 2017;8:15750

28. Pastore N, et al. TFEB and TFE3 cooperate in the regulation of the innate immune response in activated macrophages. Autophagy. 2016;12(8):1240-1258

29. Sciarretta $S$, et al. Trehalose-induced activation of autophagy improves cardiac remodeling after myocardial infarction. $J A m$ Coll Cardiol. 2018;71(18):1999-2010.

30. Sager HB, et al. Proliferation and recruitment contribute to myocardial macrophage expansion in chronic heart failure. Circ Res. 2016;119(7):853-864.

31. Kang YH, et al. Impaired macrophage autophagy induces systemic insulin resistance in obesity. Oncotarget. 2016;7(24):35577-35591.

32. Liang F, et al. Monocyte differentiation up-regulates the expression of the lysosomal sialidase, Neu1, and triggers its targeting to the plasma membrane via major histocompatibility complex class II-positive compartments. J Biol Chem. 2006;281(37):27526-27538

33. Nossuli TO, et al. A chronic mouse model of myocardial ischemia-reperfusion: essential in cytokine studies. Am J Physiol Heart Circ Physiol. 2000;278(4):H1049-H1055.

34. Lavine KJ, Kovacs A, Weinheimer C, Mann DL. Repetitive myocardial ischemia promotes coronary growth in the adult mammalian heart. J Am Heart Assoc. 2013;2(5):e000343.

35. Li L, Wang ZV, Hill JA, Lin F. New autophagy reporter mice reveal dynamics of proximal tubular autophagy. J Am Soc Nephrol. 2014;25(2):305-315.

36. Pfeffer MA, et al. Effect of captopril on mortality and morbidity in patients with left ventricular dysfunction after myocardial infarction. Results of the survival and ventricular enlargement trial. The SAVE Investigators. N Engl J Med. 1992;327(10):669-677.

37. Nahrendorf $\mathrm{M}$, et al. The healing myocardium sequentially mobilizes two monocyte subsets with divergent and complementary functions. J Exp Med. 2007;204(12):3037-3047.

38. Lavine KJ, et al. Distinct macrophage lineages contribute to disparate patterns of cardiac recovery and remodeling in the neonatal and adult heart. Proc Natl Acad Sci U S A. 2014;111(45):16029-16034.

39. Foo RS, Mani K, Kitsis RN. Death begets failure in the heart. J Clin Invest. 2005;115(3):565-571.

40. Sager HB, et al. Targeting interleukin- $1 \beta$ reduces leukocyte production after acute myocardial infarction. Circulation. 2015;132(20):1880-1890.

41. Liu J, et al. Beclin1 controls the levels of p53 by regulating the deubiquitination activity of USP10 and USP13. Cell. 2011;147(1):223-234.

42. Madisen L, et al. A robust and high-throughput Cre reporting and characterization system for the whole mouse brain. Nat Neurosci. 2010;13(1):133-140.

43. Viaud M, et al. Lysosomal cholesterol hydrolysis couples efferocytosis to anti-inflammatory oxysterol production. Circ Res 2018;122(10):1369-1384.

44. Bowden KL, et al. LAL (Lysosomal Acid Lipase) promotes reverse cholesterol transport in vitro and in vivo. Arterioscler Thromb Vasc Biol. 2018;38(5):1191-1201.

45. Westerterp M, et al. Cholesterol efflux pathways suppress inflammasome activation, NETosis, and atherogenesis. Circulation. 2018;138(9):898-912.

46. Emanuel R, et al. Induction of lysosomal biogenesis in atherosclerotic macrophages can rescue lipid-induced lysosomal dysfunction and downstream sequelae. Arterioscler Thromb Vasc Biol. 2014;34(9):1942-1952.

47. Sergin I, Razani B. Self-eating in the plaque: what macrophage autophagy reveals about atherosclerosis. Trends Endocrinol Metab. 2014;25(5):225-234.

48. Frangogiannis NG. Regulation of the inflammatory response in cardiac repair. Circ Res. 2012;110(1):159-173.

49. Lambert JM, Lopez EF, Lindsey ML. Macrophage roles following myocardial infarction. Int J Cardiol. 2008;130(2):147-158

50. Schilling JD, Machkovech HM, He L, Diwan A, Schaffer JE. TLR4 activation under lipotoxic conditions leads to synergistic macrophage cell death through a TRIF-dependent pathway. J Immunol. 2013;190(3):1285-1296.

51. Razani B, et al. Autophagy links inflammasomes to atherosclerotic progression. Cell Metab. 2012;15(4):534-544

52. Chuang SY, Yang CH, Chou CC, Chiang YP, Chuang TH, Hsu LC. TLR-induced PAI-2 expression suppresses IL-1 $\beta$ processing via increasing autophagy and NLRP3 degradation. Proc Natl Acad Sci U S A. 2013;110(40):16079-16084.

53. Weber K, Schilling JD. Lysosomes integrate metabolic-inflammatory cross-talk in primary macrophage inflammasome activation. J Biol Chem. 2014;289(13):9158-9171.

54. Claude-Taupin A, Jia J, Mudd M, Deretic V. Autophagy's secret life: secretion instead of degradation. Essays Biochem. 2017;61(6):637-647.

55. Settembre C, et al. TFEB controls cellular lipid metabolism through a starvation-induced autoregulatory loop. Nat Cell Biol. 2013;15(6):647-658

56. O'Rourke EJ, Ruvkun G. MXL-3 and HLH-30 transcriptionally link lipolysis and autophagy to nutrient availability. Nat Cell Biol. 2013;15(6):668-676.

57. Murphy JT, et al. Simple nutrients bypass the requirement for HLH-30 in coupling lysosomal nutrient sensing to survival. PLoS Biol. 2019;17(5):e3000245

58. Dang EV, McDonald JG, Russell DW, Cyster JG. Oxysterol restraint of cholesterol synthesis prevents AIM2 inflammasome activation. Cell. 2017;171(5):1057-1071.e11. 
59. Rosenbaum AI, Cosner CC, Mariani CJ, Maxfield FR, Wiest O, Helquist P. Thiadiazole carbamates: potent inhibitors of lysosomal acid lipase and potential Niemann-Pick type C disease therapeutics. J Med Chem. 2010;53(14):5281-5289.

60. House SL, Wang J, Castro AM, Weinheimer C, Kovacs A, Ornitz DM. Fibroblast growth factor 2 is an essential cardioprotective factor in a closed-chest model of cardiac ischemia-reperfusion injury. Physiol Rep. 2015;3(1):e12278.

61. Schugar RC, Moll AR, André d'Avignon D, Weinheimer CJ, Kovacs A, Crawford PA. Cardiomyocyte-specific deficiency of ketone body metabolism promotes accelerated pathological remodeling. Mol Metab. 2014;3(7):754-769.

62. Cheng SL, et al. Targeted reduction of vascular Msx1 and Msx2 mitigates arteriosclerotic calcification and aortic stiffness in LDLR-deficient mice fed diabetogenic diets. Diabetes. 2014;63(12):4326-4337.

63. Ma X, Liu H, Foyil SR, Godar RJ, Weinheimer CJ, Diwan A. Autophagy is impaired in cardiac ischemia-reperfusion injury. Autophagy. 2012;8(9):1394-1396.

64. Weber KJ, et al. PPAR $\gamma$ deficiency suppresses the release of IL-1 $\beta$ and IL-1 $\alpha$ in macrophages via a type 1 IFN-dependent mechanism. J Immunol. 2018;201(7):2054-2069. 